\title{
Prediction of Sex from Teeth Linear Dimensions and Indices
}

\author{
Hassnaa Ahmed Ahmed Ali ${ }^{1}$, Rania A. Radwan ${ }^{1}$, Maha A. Hilal ${ }^{1}$
}

\begin{tabular}{|c|c|}
\hline & ABSTRACT \\
\hline $\begin{array}{l}\text { KEYWORDS } \\
\text { Linear dimensions, } \\
\text { BL, } \\
\text { MD, } \\
\text { Dental indices, } \\
\text { Sex prediction. }\end{array}$ & $\begin{array}{l}\text { Determination of sex is one of the most important and vital steps in forensic } \\
\text { medicine. Sexual dimorphism using odontometric analysis is found to vary in } \\
\text { different populations making it necessary to attain values of a specific population } \\
\text { which makes identification of person as easy as possible. The present study aims to } \\
\text { establish sexual differentiation using dental parameters including dental linear } \\
\text { dimensions and indices. Maxillary jaw casts from total of } 100 \text { subjects (50 males } \\
\text { and } 50 \text { females) between ages } 17 \text { and } 24 \text { years were taken. Mesiodistal (MD) and } \\
\text { Buccolingual (BL) dimensions of each tooth of from maxillary jaw cast excluding } \\
\text { third molar were measured using precision calipers, dental indices were calculated. } \\
\text { Data was collected and statistical analysis was done using SPSS software version } \\
20 \text {. Bucccolingual dimension was proved to be a better parameter than MD for } \\
\text { assessment of gender in case of left central incisor, canines and left first molar. } \\
\text { Crown index of right central incisor, second premolars, and left first molars was } \\
\text { the best index for sex prediction of most of the teeth. }\end{array}$ \\
\hline
\end{tabular}

\section{Introduction}

Tooth parameters are useful methods for sex differentiation from anthropological skeletal remains. Sexual dimorphism in tooth parameters has been studied over the past halfcentury with odontologists and anthropologists (Lund and Mörnstad, 1999; İşcan and Kedici, 2003; Acharya and Mainali, 2007). Dental features in sex identification can be classified into nonmetric and metric methods. Nonmetric parameters based on the presence or absence of a particular morphological feature; shoveling of incisor, Carabelli cusps, hypocone and protostylid (Ramakrishnan et al., 2015).

\footnotetext{
${ }^{(1)}$ Forensic Medicine \& Clinical Toxicology Department, Faculty of Medicine, Sohag University. Corresponding author: 01116619059, dr_hasnaahmed@yahoo.com
}

Unlike nonmetric features, metrical methods are more structured, less subjective and it can be repeated to validate the obtained results (Bidmos et al., 2010). Linear dimension including mesio-distal and bucco-lingual and dental indices such as crown area crown module and crown index are termed metric features of the teeth (Acharya and Mainali, 2008 and Bakkannavar et al., 2012).

Many studies were conducted to determine the sex dimorphism taking a single tooth, few teeth or a quadrant into consideration. Some studies have chosen either mesiodistal or buccolingual dimensions in sex determination or single index. The present study combined these parameters for better differentiation and prediction of sexual dimorphism. 


\section{Material and methods:}

The present study was conducted on total of 100 subjects, 50 males and 50 females. The study protocol was approved by Forensic Medicine and Clinical Toxicology Department, Faculty of Medicine, Sohag University. Informed written consent was taken from subjects. Subject's age group was from 17 to 24 years old. Subjects were students' population of Sohag University, Faculty of Medicine. Visible fractures, proximal dental caries, restoration or significant attrition, subjects with braces, removable partial dentures, fixed partial dentures, cleft palate or cleft lip were excluded from the study.

Maxillary jaw casts were performed using Alginate powder, perforated metal maxillary impression tray, spatula and mixing bowl, and dental stone. Precision caliper was used for measuring of linear dimensions for all teeth except third molars.

Mesiodistal (MD) is defined as the greatest dimension between the contact points on the approximate surfaces of the crown and was measured with the caliper beaks placed occlusally along the long axis of tooth surface of the crown where it was considered that contact with adjacent teeth would have normally occurred. Buccolingual (BL) is defined as the greatest distance between the labial/buccal surface and the lingual surface of the tooth crown was measured with the caliper held at right angles to the MD dimension. Dental indices have been derived from mathematical combinations of linear measurements. Crown area is the product of $\mathrm{BL}$ and $\mathrm{MD}$ dimensions $(\mathrm{BL} \times \mathrm{MD})$. Crown module for each tooth is taken as the average of $\mathrm{BL}$ and $\mathrm{MD}$ dimensions, i.e. (BL + MD)/2.
Crown index on the other hand, is the ratio of the two linear measurements expressed as percentage, i.e. $(\mathrm{BL} / \mathrm{MD}) \times 100$ (Acharya and Mainali, 2008).

\section{Statistical analysis:}

Data was analyzed using SPSS computer program version 20. Data was expressed as median values. The data was tested for normality using Shapiro-Wilk test. The nonparametric Mann-Whitney test and Wilcoxon Signed Ranks test were used for data which was not normally distributed. Receiver operating characteristic (ROC) curve was constructed for optimum cut off points of the studied measures in predicting male gender and the area under the ROC curve value with 95\% CI was calculated. Optimal cut-off values were determined; sensitivity, specificity, positive predictive value, negative predictive value were calculated. Logistic regression test was used to determine the role of the studied measures in predicting male gender. A 5\% level was chosen as a level of significance in all statistical tests used in the study.

\section{Results}

\section{Central incisor}

There was significant statistical increase in median value of right central incisor's (BL, crown area and crown index) and left central incisor (BL, crown area, crown index and crown module) in males than females, see median and $p$-values in table (1) . 
Table (1): Comparison of the median values of central incisors dental parameters between males and females $(n=100)$

\begin{tabular}{|c|c|c|c|c|c|c|}
\hline \multirow{3}{*}{ Variables } & \multicolumn{3}{|c|}{ Right central incisor } & \multicolumn{3}{|c|}{ Left central incisor } \\
\hline & \multicolumn{2}{|c|}{ Median } & \multirow[b]{2}{*}{ p-value } & \multicolumn{2}{|c|}{ Median } & \multirow[b]{2}{*}{ p-value } \\
\hline & $\begin{array}{l}\text { Males } \\
(\mathrm{n}=50)\end{array}$ & $\begin{array}{c}\text { Females } \\
(n=50)\end{array}$ & & $\begin{array}{l}\text { Males } \\
(\mathrm{n}=50)\end{array}$ & $\begin{array}{c}\text { Females } \\
(n=50)\end{array}$ & \\
\hline MD & 8.5 & 8.5 & 0.523 & 8.2 & 8.5 & 0.111 \\
\hline$\overline{\mathrm{BL}}$ & 4.2 & 4 & $<0.001^{*}$ & 4.5 & 3.6 & $0.003^{*}$ \\
\hline Crown area & 34.86 & 32.3 & $0.045^{*}$ & 38.25 & 33.25 & $0.01^{*}$ \\
\hline Crown module & 6.25 & 6.15 & 0.149 & 6.5 & 6.25 & $0.026^{*}$ \\
\hline Crown index $\%$ & 50.6 & 46.15 & $0.003^{*}$ & 50 & 44.44 & $0.003^{*}$ \\
\hline
\end{tabular}

MD: mesiodistal dimension, BL: buccolingual dimension, $p$-value is calculated by Mann-Whitney $U$ test, $p$-value $<0.05$ is statistically significant.

By using ROC curve, BL, crown area and crown index of the right central incisor had significant predicting value for male gender. Buccolingual dimension, crown area, crown module and crown index of left central incisor had significant predicting value for male gender, see AUC areas and cut off points in table (2).

Table (2): Receiver operating characteristic (ROC) curve of central incisor measures for optimum cutoff points in predicting male gender.

\begin{tabular}{|c|c|c|c|c|c|c|c|c|c|c|c|c|c|c|}
\hline \multirow[b]{2}{*}{ Marker } & \multicolumn{7}{|c|}{ Right central incisor } & \multicolumn{7}{|c|}{ Left central incisor } \\
\hline & Cutoff & AUC & $\begin{array}{l}\text { Sens } \\
(\%)\end{array}$ & $\begin{array}{c}\text { Spec } \\
(\%)\end{array}$ & $\begin{array}{l}\text { PPV } \\
(\%)\end{array}$ & $\begin{array}{l}\text { NPV } \\
(\%)\end{array}$ & p-value & Cutoff & AUC & $\begin{array}{l}\text { Sens } \\
(\%)\end{array}$ & $\begin{array}{c}\text { Spec } \\
(\%)\end{array}$ & $\begin{array}{l}\text { PPV } \\
(\%)\end{array}$ & $\begin{array}{l}\text { NPV } \\
(\%)\end{array}$ & p-value \\
\hline MD & $\leq 7.2$ & 0.54 & 24 & 100 & 100 & 56.8 & 0.539 & $\leq 7.6$ & 0.59 & 24 & 100 & 100 & 56.8 & 0.116 \\
\hline$\overline{\mathrm{BL}}$ & $>4$ & 0.74 & 60 & 88 & 83.3 & 68.7 & $<0.001^{*}$ & $>4$ & 0.668 & 56 & 80 & 73.7 & 64.5 & $0.003 *$ \\
\hline Crown area & $>32.3$ & 0.62 & 68 & 64 & 65.4 & 66.7 & $0.045^{*}$ & $>36$ & 0.65 & 52 & 92 & 86.7 & 65.7 & $0.011 *$ \\
\hline $\begin{array}{l}\text { Crown } \\
\text { module }\end{array}$ & $>6.5$ & 0.58 & 28 & 100 & 100 & 58.1 & 0.157 & $>6.52$ & 0.627 & 40 & 100 & 100 & 62.5 & $0.03^{*}$ \\
\hline Crown index & $>53.33$ & 0.67 & 44 & 100 & 100 & 64.1 & $0.002 *$ & $>47.06$ & 0.672 & 52 & 80 & 72.2 & 62.5 & $0.002 *$ \\
\hline
\end{tabular}

MD: mesiodistal dimension, BL: buccolingual dimension, Sens: sensitivity, spec: specificity, AUC: area under curve, PPV: positive predictive value, NPV: negative predictive value.

\section{Lateral incisor}

There was significant statistical increase in median value of right lateral incisor's (MD, BL, crown area and crown module) and of left lateral incisor's (MD, BL, crown area, crown module and crown index) in males than females. See median and p-values in table (3).

Table (3): Comparison of the median values of lateral incisors dental parameters between males and females $(\mathrm{n}=100)$.

\begin{tabular}{|c|c|c|c|c|c|c|}
\hline \multirow{3}{*}{ Variables } & \multicolumn{3}{|c|}{ Right lateral incisor } & \multicolumn{3}{|c|}{ Left lateral incisor } \\
\hline & \multicolumn{2}{|c|}{ Median } & \multirow[b]{2}{*}{ p-value } & \multicolumn{2}{|c|}{ Median } & \multirow[b]{2}{*}{ p-value } \\
\hline & $\begin{array}{l}\text { Males } \\
(\mathrm{n}=50)\end{array}$ & $\begin{array}{c}\text { Females } \\
(n=50)\end{array}$ & & $\begin{array}{l}\text { Males } \\
(\mathrm{n}=50)\end{array}$ & $\begin{array}{c}\text { Females } \\
(\mathbf{n}=\mathbf{5 0})\end{array}$ & \\
\hline $\mathrm{MD}$ & 6.6 & 6.3 & 0.011 & 6.7 & 6.2 & $0.009 *$ \\
\hline $\mathrm{BL}$ & 4 & 3.6 & 0.003 & 4.2 & 3.8 & $<0.001^{*}$ \\
\hline Crown area & 26.66 & 22.8 & $<0.001$ & 28.14 & 23.56 & $<0.001^{*}$ \\
\hline Crown module & 5.25 & 4.9 & $<0.001$ & 5.45 & 5 & $<0.001^{*}$ \\
\hline Crown index\% & 57.97 & 58.33 & 0.281 & 62.69 & 60.56 & $0.028 *$ \\
\hline
\end{tabular}

MD: mesiodistal dimension, BL: buccolingual dimension, p-value is calculated by Mann-Whitney $U$ test, $p$-value $<0.05$ is statistically significant. 
By using ROC curve, MD, BL, crown area and crown module of right lateral incisor and left lateral incisor had significant predicting value for male gender. Mesiodistal dimension, BL, crown area, crown module and crown index of left lateral incisor. See AUC areas and cut off points in table (4).

Table (4): Receiver operating characteristic (ROC) curve of lateral incisor measures for optimum cut off points in predicting male gender.

\begin{tabular}{|c|c|c|c|c|c|c|c|c|c|c|c|c|c|c|}
\hline \multirow[b]{2}{*}{ Marker } & \multicolumn{7}{|c|}{ Right lateral incisor } & \multicolumn{7}{|c|}{ Left lateral incisor } \\
\hline & Cutoff & AUC & $\begin{array}{l}\text { Sens } \\
(\%) \\
\end{array}$ & $\begin{array}{c}\text { Spec } \\
(\%)\end{array}$ & $\begin{array}{l}\text { PPV } \\
(\%)\end{array}$ & $\begin{array}{l}\text { NPV } \\
(\%)\end{array}$ & p-value & Cutoff & AUC & $\begin{array}{l}\text { Sens } \\
(\%)\end{array}$ & $\begin{array}{c}\text { Spec } \\
(\%)\end{array}$ & $\begin{array}{l}\text { PPV } \\
(\%) \\
\end{array}$ & $\begin{array}{l}\text { NPV } \\
(\%) \\
\end{array}$ & p-value \\
\hline MD & $>6$ & 0.646 & 76 & 60 & 65.5 & 71.4 & $0.009 *$ & $>6.5$ & 0.650 & 64 & 84 & 70 & 48 & $0.011^{*}$ \\
\hline $\mathrm{BL}$ & $>3.8$ & 0.672 & 68 & 92 & 89.5 & 74.2 & $0.009 *$ & $>4$ & 0.768 & 68 & 84 & 72.4 & 52 & $<0.001^{*}$ \\
\hline Crown area & $>24.85$ & 0.750 & 68 & 100 & 100 & 75.8 & $<0.001 *$ & $>24$ & 0.802 & 84 & 76 & 82.6 & 60 & $<0.001 *$ \\
\hline Crown module & $>5.3$ & 0.724 & 48 & 100 & 100 & 65.8 & $<0.001^{*}$ & $>5$ & 0.755 & 76 & 76 & 76 & 52 & $<0.001^{*}$ \\
\hline Crown index & $>66.67$ & 0.562 & 32 & 92 & 80 & 57.5 & 0.291 & $>61.29$ & 0.627 & 64 & 72 & 66.7 & 36 & $0.026^{*}$ \\
\hline
\end{tabular}

MD: mesiodistal dimension, BL: buccolingual dimension, Sens: sensitivity, spec: specificity, AUC: area under curve, PPV: positive predictive value, NPV: negative predictive value

\section{Canines}

There was significant statistical increase in median value of right canine's MD, left canine's crown area, crown module in males than females .There was significant statistical decrease in median value of right canines, BL, crown area and crown index in males than females. See median and p-values in table (5).

Table (5): Comparison of the median values of canine dental parameters between males and females $(n=100)$

\begin{tabular}{|c|c|c|c|c|c|c|}
\hline \multirow{3}{*}{ Variables } & \multicolumn{3}{|c|}{ Right canine } & \multicolumn{3}{|c|}{ Left canine } \\
\hline & \multicolumn{2}{|c|}{ Median } & \multirow[b]{2}{*}{ p-value } & \multicolumn{2}{|c|}{ Median } & \multirow[b]{2}{*}{ p-value } \\
\hline & $\begin{array}{l}\text { Males } \\
(\mathrm{n}=50)\end{array}$ & $\begin{array}{c}\text { Females } \\
(n=50)\end{array}$ & & $\begin{array}{l}\text { Males } \\
(\mathrm{n}=50)\end{array}$ & $\begin{array}{c}\text { Females } \\
(n=50)\end{array}$ & \\
\hline $\mathrm{MD}$ & 7.3 & 7 & $0.001 *$ & 7 & 7 & 0.107 \\
\hline $\mathrm{BL}$ & 5 & 5.5 & $<0.001^{*}$ & 5.5 & 5 & 0.077 \\
\hline Crown area & 36 & 37.8 & $0.051 *$ & 38.4 & 33 & $<0.001^{*}$ \\
\hline Crown module & 6.25 & 6.2 & 0.157 & 6.4 & 5.9 & $<0.001^{*}$ \\
\hline Crown index $\%$ & 64.79 & 78.57 & $<0.001^{*}$ & 83.1 & 71.43 & 0.836 \\
\hline
\end{tabular}

MD: mesiodistal dimension, BL: buccolingual dimension, $p$-value is calculated by Mann-Whitney $U$ test, $p$-value $<0.05$ is statistically significant.

By using ROC curve MD, BL, crown area and crown index of right canine had significant predicting value for male gender, crown area and crown module of the left canine had significant predicting value for male gender. See AUC areas and cut off points table in (6).

Table (6): Receiver operating characteristic (ROC) curve of canine measures for optimum cut off points in predicting male gender.

\begin{tabular}{|c|c|c|c|c|c|c|c|c|c|c|c|c|c|c|}
\hline \multirow[b]{2}{*}{ Marker } & \multicolumn{7}{|c|}{ Right canine } & \multicolumn{7}{|c|}{ Left canine } \\
\hline & Cutoff & AUC & $\begin{array}{l}\text { Sens } \\
(\%)\end{array}$ & $\begin{array}{c}\text { Spec } \\
(\%)\end{array}$ & $\begin{array}{l}\text { PPV } \\
(\%)\end{array}$ & $\begin{array}{l}\text { NPV } \\
(\%)\end{array}$ & P-value & Cutoff & AUC & $\begin{array}{l}\text { Sens } \\
(\%)\end{array}$ & $\begin{array}{c}\text { Spec } \\
(\%)\end{array}$ & $\begin{array}{l}\text { PPV } \\
(\%)\end{array}$ & $\begin{array}{l}\text { NPV } \\
(\%)\end{array}$ & P-value \\
\hline MD & $>7$ & 0.690 & 68 & 64 & 65.4 & 66.7 & 0.003 & $>7.5$ & 0.593 & 32 & 100 & 59.5 & 32 & 0.112 \\
\hline $\mathrm{BL}$ & $\leq 4.6$ & 0.728 & 48 & 100 & 100 & 65.8 & $<0.001^{*}$ & $>5.2$ & 0.602 & 64 & 68 & 65.4 & 32 & 0.079 \\
\hline Crown area & $\leq 34.04$ & 0.613 & 32 & 100 & 100 & 59.5 & 0.051 & $>35$ & 0.714 & 84 & 68 & 81 & 52 & $0.002^{*}$ \\
\hline Crown module & $\leq 7.15$ & 0.581 & 96 & 32 & 58.5 & 88.9 & 0.169 & $>6$ & 0.714 & 76 & 76 & 76 & 52 & $0.002^{*}$ \\
\hline Crown index & $\leq 64.79$ & 0.779 & 56 & 100 & 100 & 69.4 & $<0.001^{*}$ & $>61.64$ & 0.512 & 84 & 32 & 66.7 & 16 & 0.839 \\
\hline
\end{tabular}

MD: mesiodistal dimension, BL: buccolingual dimension, Sens: sensitivity, spec: specificity, AUC: area under curve, PPV: positive predictive value, NPV: negative predictive value. 


\section{Premolars}

\section{First premolar}

There was statistical significant increase in median value, of left first premolar's MD and statistical significant decrease in crown index between males and females. See median and $p$-values in table (7).

Table (7): Comparison of the median values of first premolar dental parameters between males and females $(n=100)$.

\begin{tabular}{|c|c|c|c|c|c|c|}
\hline \multirow{3}{*}{ Variables } & \multicolumn{3}{|c|}{ Right first premolar } & \multicolumn{3}{|c|}{ Left first premolar } \\
\hline & \multicolumn{2}{|c|}{ Median } & \multirow[b]{2}{*}{$\mathrm{p}$-value } & \multicolumn{2}{|c|}{ Median } & \multirow[b]{2}{*}{ p-value } \\
\hline & $\begin{array}{l}\text { Males } \\
(\mathrm{n}=50)\end{array}$ & $\begin{array}{c}\text { Females } \\
(\mathrm{n}=50)\end{array}$ & & $\begin{array}{l}\text { Males } \\
(\mathrm{n}=50)\end{array}$ & $\begin{array}{c}\text { Females } \\
(\mathrm{n}=50)\end{array}$ & \\
\hline $\mathrm{MD}$ & 6.5 & 6.3 & 0.097 & 7 & 7 & $0.022^{*}$ \\
\hline BL & 8.5 & 8.5 & 0.9 & 9 & 8.9 & 0.076 \\
\hline Crown area & 55.04 & 55.25 & 0.431 & 62.1 & 61 & 0.118 \\
\hline Crown module & 7.5 & 7.5 & 0.454 & 7.95 & 7.85 & 0.112 \\
\hline Crown index \% & 129.33 & 133.33 & 0.638 & 126.58 & 128.57 & $0.007^{*}$ \\
\hline
\end{tabular}

MD: mesiodistal dimension, BL: buccolingual dimension, p-value is calculated by Mann-Whitney U test, $\mathrm{p}$-value $<0.05$ is statistically significant.

By using ROC curve MD and crown index of the left first premolar had significant predicting value for male gender. See AUC areas and cut off points in table (8).

Table (8): Receiver operating characteristic (ROC) curve of first premolar measures for optimum cutoff points in predicting male gender.

\begin{tabular}{|c|c|c|c|c|c|c|c|c|c|c|c|c|c|c|}
\hline \multirow[b]{2}{*}{ Marker } & \multicolumn{7}{|c|}{ Right first premolar } & \multicolumn{7}{|c|}{ Left first premolar } \\
\hline & Cutoff & AUC & $\begin{array}{l}\text { Sens } \\
(\%) \\
\end{array}$ & $\begin{array}{l}\text { Spec } \\
(\%)\end{array}$ & $\begin{array}{l}\text { PPV } \\
(\%) \\
\end{array}$ & $\begin{array}{l}\text { NPV } \\
(\%) \\
\end{array}$ & $\begin{array}{c}\text { P- } \\
\text { value }\end{array}$ & Cutoff & AUC & $\begin{array}{l}\text { Sens } \\
(\%)\end{array}$ & $\begin{array}{l}\text { Spec } \\
(\%)\end{array}$ & $\begin{array}{l}\text { PPV } \\
(\%) \\
\end{array}$ & $\begin{array}{l}\text { NPV } \\
(\%) \\
\end{array}$ & $\begin{array}{c}\begin{array}{c}P- \\
\text { value }\end{array} \\
\end{array}$ \\
\hline MD & $>6.7$ & 0.595 & 48 & 80 & 70.6 & 60.6 & 0.099 & $>7.3$ & 0.632 & 44 & 100 & 100 & 64.1 & $0.023^{*}$ \\
\hline $\mathrm{BL}$ & $>9$ & 0.507 & 20 & 100 & 100 & 55.6 & 0.903 & $>9$ & 0.507 & 20 & 100 & 100 & 55.6 & 0.903 \\
\hline $\begin{array}{l}\text { Crown } \\
\text { area }\end{array}$ & $>67.5$ & 0.546 & 20 & 100 & 100 & 55.6 & 0.435 & $>64.8$ & 0.59 & 48 & 100 & 100 & 65.8 & 0.147 \\
\hline $\begin{array}{l}\text { Crown } \\
\text { module }\end{array}$ & $>8.25$ & 0.543 & 20 & 100 & 100 & 55.6 & 0.459 & $>8.1$ & 0.592 & 48 & 100 & 100 & 65.8 & 0.139 \\
\hline $\begin{array}{l}\text { Crown } \\
\text { index }\end{array}$ & $\leq 129.33$ & 0.527 & 52 & 80 & 72.2 & 62.5 & 0.652 & $\leq 127.87$ & 0.656 & 68 & 72 & 70.8 & 69.2 & $0.006^{*}$ \\
\hline
\end{tabular}

MD: mesiodistal dimension, BL: buccolingual dimension, Sens: sensitivity, spec: specificity, AUC: area under curve, PPV: positive predictive value, NPV: negative predictive value.

\section{Second premolars}

There was significant statistical increase in median value of right second premolar's $\mathrm{MD}, \mathrm{BL}$, crown area and crown module and significant decrease in median value of crown index and left second premolar's crown index in males than females. See median and p-values table in (9). 
Table (9): Comparison of the median values of second premolars dental parameters between males and females $(n=100)$.

\begin{tabular}{|c|c|c|c|c|c|c|}
\hline \multirow{3}{*}{ Variables } & \multicolumn{3}{|c|}{ Right second premolar } & \multicolumn{3}{|c|}{ Left second premolar } \\
\hline & \multicolumn{2}{|c|}{ Median } & \multirow[b]{2}{*}{ p-value } & \multicolumn{2}{|c|}{ Median } & \multirow[b]{2}{*}{ p-value } \\
\hline & $\begin{array}{l}\text { Males } \\
(\mathrm{n}=\mathbf{5 0})\end{array}$ & $\begin{array}{c}\text { Females } \\
(n=50)\end{array}$ & & $\begin{array}{l}\text { Males } \\
(n=50)\end{array}$ & $\begin{array}{c}\text { Females } \\
(n=50)\end{array}$ & \\
\hline $\mathrm{MD}$ & 7 & 5.4 & $<0.001^{*}$ & 6.7 & 6.5 & 0.317 \\
\hline $\mathrm{BL}$ & 9.5 & 9 & $<0.001^{*}$ & 9.2 & 9 & 0.072 \\
\hline Crown area & 63.7 & 47.5 & $<0.001^{*}$ & 62.78 & 58.5 & 0.525 \\
\hline Crown module & 8.15 & 7.25 & $<0.001^{*}$ & 7.95 & 7.75 & 0.524 \\
\hline Crown index $\%$ & 130.88 & 157.41 & $<0.001^{*}$ & 128.57 & 138.46 & $0.001 *$ \\
\hline
\end{tabular}

MD: mesiodistal dimension, BL: buccolingual dimension, $\mathrm{p}$-value is calculated by Mann-Whitney $\mathrm{U}$ test, $\mathrm{p}$-value $<0.05$ is statistically significant .

By using ROC curve, MD, BL, crown area, crown module and crown index of the right second premolar, crown index of left second premolar had significant predicting value for male gender. See AUC areas and cut off points in table (10).

Table (10): Receiver operating characteristic (ROC) curve of second premolar measures for optimum cut off points in predicting male gender.

\begin{tabular}{|c|c|c|c|c|c|c|c|c|c|c|c|c|c|c|}
\hline \multirow[b]{2}{*}{ Marker } & \multicolumn{7}{|c|}{ Right second premolar } & \multicolumn{7}{|c|}{ Left second premolar } \\
\hline & Cutoff & AUC & $\begin{array}{l}\text { Sens } \\
(\%)\end{array}$ & $\begin{array}{l}\text { Spec } \\
(\%)\end{array}$ & $\begin{array}{l}\text { PPV } \\
(\%)\end{array}$ & $\begin{array}{l}\text { NPV } \\
(\%)\end{array}$ & P-value & Cutoff & $\mathbf{A U C}$ & $\begin{array}{l}\text { Sens } \\
(\%)\end{array}$ & $\begin{array}{l}\text { Spec } \\
(\%)\end{array}$ & $\begin{array}{l}\text { PPV } \\
(\%)\end{array}$ & $\begin{array}{l}\text { NPV } \\
(\%)\end{array}$ & $\begin{array}{c}\text { P- } \\
\text { value }\end{array}$ \\
\hline MD & $>6.2$ & 0.880 & 76 & 92 & 90.5 & 79.3 & $<0.001^{*}$ & $>6.59$ & 0.558 & 52 & 76 & 68.4 & 61.3 & 0.326 \\
\hline $\mathrm{BL}$ & $>9.5$ & 0.702 & 40 & 92 & 83.3 & 60.5 & $0.001 *$ & $\leq 8.6$ & 0.602 & 40 & 100 & 100 & 62.5 & 0.082 \\
\hline $\begin{array}{l}\text { Crown } \\
\text { area }\end{array}$ & $>54$ & 0.856 & 84 & 92 & 91.3 & 85.2 & $<0.001 *$ & $>59.31$ & 0.537 & 64 & 68 & 66.7 & 65.4 & 0.547 \\
\hline $\begin{array}{l}\text { Crown } \\
\text { module }\end{array}$ & $>7.5$ & 0.837 & 84 & 92 & 91.3 & 85.2 & $<0.001^{*}$ & $>7.85$ & 0.535 & 64 & 68 & 66.7 & 65.4 & 0.565 \\
\hline $\begin{array}{l}\text { Crown } \\
\text { index }\end{array}$ & $\leq 154.55$ & 0.810 & 92 & 56 & 67.6 & 87.5 & $<0.001 *$ & $\leq 133.33$ & 0.686 & 68 & 76 & 73.9 & 70.4 & $0.001 *$ \\
\hline
\end{tabular}

MD: mesiodistal dimension, BL: buccolingual dimension, Sens: sensitivity, spec: specificity, AUC: area under curve, PPV: positive predictive value, NPV: negative predictive value.

\section{Molars}

\section{First molar}

There was statistical significant increase in median value of right first molar's MD, crown area and crown module and significant decrease in median value of BL and crown index of right first molar and BL of left first molar in male than female See median and pvalues in table (11). 
Table (11): Comparison of the median values of first molar dental parameters between males and females $(n=100)$.

\begin{tabular}{|c|c|c|c|c|c|c|}
\hline \multirow{3}{*}{ Variables } & \multicolumn{3}{|c|}{ Right first molar } & \multicolumn{3}{|c|}{ Left first molar } \\
\hline & \multicolumn{2}{|c|}{ Median } & \multirow[b]{2}{*}{ p-value } & \multicolumn{2}{|c|}{ Median } & \multirow[b]{2}{*}{ p-value } \\
\hline & $\begin{array}{l}\text { Males } \\
(n=50)\end{array}$ & $\begin{array}{c}\text { Females } \\
(n=50)\end{array}$ & & $\begin{array}{l}\text { Males } \\
(\mathrm{n}=\mathbf{5 0})\end{array}$ & $\begin{array}{c}\text { Females } \\
(n=50)\end{array}$ & \\
\hline $\mathrm{MD}$ & 9.3 & 9 & $<0.001$ & 9.4 & 9.5 & 0.703 \\
\hline $\mathrm{BL}$ & 9.9 & 10 & 0.004 & 9.8 & 10 & $0.002 *$ \\
\hline Crown area & 91.14 & 90 & 0.009 & 90.25 & 95 & 0.381 \\
\hline Crown module & 9.55 & 9.5 & 0.02 & 9.5 & 9.75 & 0.337 \\
\hline Crown index $\%$ & 105.26 & 111.11 & $<0.001$ & 100 & 105.26 & 0.245 \\
\hline
\end{tabular}

MD: mesiodistal dimension, BL: buccolingual dimension, $\mathrm{p}$-value is calculated by Mann-Whitney $\mathrm{U}$ test, $\mathrm{p}$-value $<0.05$ is statistically significant.

By using ROC curve, MD, BL, crown area, crown module and crown index of right first molar had significant predicting value for male gender. Buccolingual dimension of first molar had significant predicting value for male gender. See AUC areas and cut off points in table (12).

Table (12): Receiver operating characteristic (ROC) curve of first molar measures for optimum cut off points in predicting male gender.

\begin{tabular}{|c|c|c|c|c|c|c|c|c|c|c|c|c|c|c|}
\hline \multirow[b]{2}{*}{ Marker } & \multicolumn{7}{|c|}{ Right first molar } & \multicolumn{7}{|c|}{ Left first molar } \\
\hline & Cutoff & AUC & $\begin{array}{l}\text { Sens } \\
(\%)\end{array}$ & $\begin{array}{c}\text { Spec } \\
(\%)\end{array}$ & $\begin{array}{l}\text { PPV } \\
(\%)\end{array}$ & $\begin{array}{l}\text { NPV } \\
(\%)\end{array}$ & p-value & Cutoff & AUC & $\begin{array}{c}\text { Sens } \\
(\%)\end{array}$ & $\begin{array}{c}\text { Spec } \\
(\%)\end{array}$ & $\begin{array}{l}\text { PPV } \\
(\%)\end{array}$ & $\begin{array}{l}\text { NPV } \\
(\%)\end{array}$ & $\begin{array}{c}\text { p- } \\
\text { value }\end{array}$ \\
\hline MD & $>9$ & 0.716 & 76 & 80 & 79.2 & 76.9 & 0.001 & $\leq 8.5$ & 0.522 & 28 & 80 & 58.3 & 52.6 & 0.711 \\
\hline $\mathrm{BL}$ & $\leq 9.9$ & 0.650 & 56 & 72 & 66.7 & 62.1 & $0.007^{*}$ & $\leq 9.5$ & 0.656 & 44 & 92 & 84.6 & 62.2 & $0.005^{*}$ \\
\hline Crown area & $>90$ & 0.650 & 60 & 80 & 75 & 66.7 & $0.008^{*}$ & $\leq 83.3$ & 0.550 & 44 & 92 & 84.6 & 62.2 & 0.405 \\
\hline Crown module & $>9.5$ & 0.634 & 60 & 80 & 75 & 66.7 & $0.019^{*}$ & $\leq 9.15$ & 0.555 & 44 & 92 & 84.6 & 62.2 & 0.362 \\
\hline Crown index & $\leq 110$ & 0.759 & 92 & 68 & 74.2 & 89.5 & $<0.001 *$ & $\leq 101.01$ & 0.566 & 60 & 64 & 62.2 & 61.5 & 0.256 \\
\hline
\end{tabular}

MD: mesiodistal dimension, BL: buccolingual dimension, Sens: sensitivity, spec: specificity, AUC: area under curve, PPV: positive predictive value, NPV: negative predictive value.

\section{Second molars}

There was statistical significant difference in median value of right second molar's MD and BL, statistical significant increase median value right second molar's crown area, significant decrease in median value of crown index in males than females. See median and p-values in table (13).

Table (13): Comparison of the median values of second molars dental parameters between males and females $(n=100)$.

\begin{tabular}{|c|c|c|c|c|c|c|}
\hline \multirow{3}{*}{ Variables } & \multicolumn{3}{|c|}{ Right second molar } & \multicolumn{3}{|c|}{ Left second molar } \\
\hline & \multicolumn{2}{|c|}{ Median } & \multirow[b]{2}{*}{ p-value } & \multicolumn{2}{|c|}{ Median } & \multirow[b]{2}{*}{ p-value } \\
\hline & $\begin{array}{l}\text { Males } \\
(n=50)\end{array}$ & $\begin{array}{c}\text { Females } \\
(n=50)\end{array}$ & & $\begin{array}{l}\text { Males } \\
(n=50)\end{array}$ & $\begin{array}{c}\text { Females } \\
(n=50)\end{array}$ & \\
\hline MD & 9 & 9 & $0.011^{*}$ & 9.3 & 9 & 0.412 \\
\hline $\mathrm{BL}$ & 10 & 10 & $<0.001 *$ & 10 & 9.8 & 0.725 \\
\hline Crown area & 91.2 & 90 & 0.023 & 90 & 90 & 0.325 \\
\hline Crown module & 9.5 & 9.5 & 0.134 & 9.5 & 9.5 & 0.325 \\
\hline Crown index\% & 106.25 & 111.11 & $<0.001 *$ & 105.26 & 107.78 & 0.771 \\
\hline
\end{tabular}

MD: mesiodistal dimension, BL: buccolingual dimension, $\mathrm{p}$-value is calculated by Mann-Whitney $\mathrm{U}$ test, $\mathrm{p}$-value $<0.05$ is statistically significant . 
By using ROC curve MD, BL, crown area, and crown index of right second molar had significant predicting value for male gender. See AUC areas and cut off points in table (14).

Table (14): Receiver operating characteristic (ROC) curve of second molar measures for optimum cut off points in predicting male gender.

\begin{tabular}{|c|c|c|c|c|c|c|c|c|c|c|c|c|c|c|}
\hline \multirow[b]{2}{*}{ Marker } & \multicolumn{7}{|c|}{ Right second molar } & \multicolumn{7}{|c|}{ Left second molar } \\
\hline & Cutoff & AUC & $\begin{array}{c}\text { Sens } \\
(\%)\end{array}$ & $\begin{array}{l}\begin{array}{l}\text { Spec } \\
(\%)\end{array} \\
\end{array}$ & $\begin{array}{r}\text { PPV } \\
(\%) \\
\end{array}$ & $\begin{array}{l}\text { NPV } \\
(\%)\end{array}$ & p-value & Cutoff & AUC & $\begin{array}{l}\text { Sens } \\
(\%)\end{array}$ & $\begin{array}{c}\text { Spec } \\
(\%)\end{array}$ & $\begin{array}{l}\text { PPV } \\
(\%)\end{array}$ & $\begin{array}{c}\text { NPV } \\
(\%)\end{array}$ & $\begin{array}{c}\mathrm{p}- \\
\text { value }\end{array}$ \\
\hline $\mathrm{MD}$ & $>9.3$ & 0.646 & 48 & 100 & 100 & 65.8 & 0.011 & $\leq 9.5$ & 0.546 & 80 & 48 & 60.6 & 70.6 & 0.435 \\
\hline $\mathrm{BL}$ & $\leq 9.7$ & 0.696 & 40 & 100 & 100 & 62.5 & $0.002 *$ & $>9$ & 0.519 & 72 & 0 & 41.9 & 0 & 0.75 \\
\hline $\begin{array}{l}\text { Crown } \\
\text { area }\end{array}$ & $>93$ & 0.630 & 36 & 100 & 100 & 61 & $0.024 *$ & $\leq 83.7$ & 0.557 & 40 & 80 & 76.9 & 59.5 & 0.334 \\
\hline $\begin{array}{l}\text { Crown } \\
\text { module }\end{array}$ & $>9.65$ & 0.586 & 40 & 100 & 100 & 62.5 & 0.149 & $\leq 9.15$ & 0.557 & 40 & 80 & 76.9 & 59.5 & 0.334 \\
\hline $\begin{array}{l}\text { Crown } \\
\text { index }\end{array}$ & $\leq 106.25$ & 0.753 & 56 & 100 & 100 & 64.1 & $<0.001 *$ & $\leq 106.38$ & 0.517 & 72 & 52 & 60 & 65 & 0.777 \\
\hline
\end{tabular}

MD: mesiodistal dimension, BL: buccolingual dimension, Sens: sensitivity, spec: specificity AUC: area under curve, PPV: positive predictive value, NPV: negative predictive value.

By the use of multiple binary logistic regression analysis and calculation of odds ratio (OR) at confidence interval (CI) 95\%, it had been concluded that the teeth parameter seen in table (15) were the best parameters in predicting of male gender.

Table (15): Final model of multiple binary logistic regression analysis about measures predicting of male gender.

\begin{tabular}{|c|c|c|c|c|c|}
\hline Variables & Adjusted OR & $\mathrm{p}$ - value & Variables & Adjusted OR & $\mathrm{p}-$ value \\
\hline \multicolumn{3}{|c|}{ Right central incisor } & \multicolumn{3}{|c|}{ Left central incisor } \\
\hline Crown index & $1.11(1.04-1.2)$ & $0.001 *$ & \multirow{2}{*}{$\mathrm{BL}$} & \multirow{2}{*}{$7.49(1.13-49.66)$} & \multirow{2}{*}{$0.037 *$} \\
\hline Crown area & $1.12(1.02-1.22)$ & $0.018^{*}$ & & & \\
\hline \multicolumn{3}{|c|}{ Right lateral incisor } & \multicolumn{3}{|c|}{ Left lateral incisor } \\
\hline Crown area & $1.26(1.1-1.44)$ & $0.001 *$ & \multirow{2}{*}{$\begin{array}{c}\text { Crown } \\
\text { area }\end{array}$} & \multirow{2}{*}{$1.26(1.1-1.44)$} & \multirow{2}{*}{$0.001 *$} \\
\hline Crown area & $1.26(1.1-1.44)$ & $0.001 *$ & & & \\
\hline \multicolumn{3}{|c|}{ Right canine } & \multicolumn{3}{|c|}{ Left canine } \\
\hline $\mathrm{MD}$ & $10.62(2.94-38.45)$ & $<0.001^{*}$ & \multirow{2}{*}{ MD } & \multirow{2}{*}{$5.57(1.08-28.81)$} & \multirow{2}{*}{$0.04 *$} \\
\hline Crown area & $0.89(0.83-0.95)$ & $0.001 *$ & & & \\
\hline \multicolumn{3}{|c|}{ Right second premolar } & \multicolumn{3}{|c|}{ Left second premolar } \\
\hline Crown index & $0.93(0.91-0.96)$ & $<0.001 *$ & $\begin{array}{l}\text { Crown } \\
\text { index }\end{array}$ & $0.96(0.93-0.99)$ & $0.004 *$ \\
\hline $\mathrm{BL}$ & $5.34(2.04-13.99)$ & $0.001 *$ & $\mathrm{BL}$ & $0.39(0.18-0.83)$ & $0.014^{*}$ \\
\hline \multicolumn{3}{|c|}{ Right first molar } & \multicolumn{3}{|c|}{ Left first molar } \\
\hline Crown index & $0.89(0.79-0.99)$ & $<0.001 *$ & \multirow{2}{*}{$\mathrm{BL}$} & \multirow{2}{*}{$0.33(0.12-0.88)$} & \multirow{2}{*}{$0.027^{*}$} \\
\hline Crown area & $0.79(0.7-0.89)$ & $0.035 *$ & & & \\
\hline \multicolumn{3}{|c|}{ Right second molar } & \multicolumn{3}{|c|}{ Left second molar } \\
\hline $\mathrm{MD}$ & $29.11(6.69-126.62)$ & $<0.001 *$ & & & \\
\hline
\end{tabular}

OD: odds ratio, ${ }^{*}$ statistically significant, (CI $\left.95 \%\right)$ : confidence interval , MD: mesiodistal dimension, BL: buccolingual dimension. 


\section{Discussion}

Findings of central incisor linear dimensions in the present study were in agreement with the studies done by Srivastava et al. (2014); Sabóia et al. (2013) and Nahidh, (2014) where they proved that sexual dimorphism is more pronounced in (BL) than in (MD) diameters. Zorba et al. (2011) noted that BL dimensions were more dimorphic than (MD) diameters in most populations. In contrast to the current study, Astete et al. (2009) proved that (MD) is better sex predictor.

Lateral incisor results of the current study were in agreement with Kailash et al. (2018) who detected statistical significance differences in $\mathrm{BL}$ and MD dimensions of the left and right lateral incisors. In contrast, Babu et al. (2016) and Srinivasprasad et al. (2017) found that lateral incisor dimensions didn't show any statistical significant differences between males and females.

Acharya and Mainali (2007); Omar and Azab (2009); Pereira et al. (2010) Zorba et al. (2011) as well as Staka and Bibmbashi (2013) founded that there was statistical significant differences between males and females in MD and BL dimensions of canines, which correlates with the current study. In contrast, Al-Rifaiy et al. (1997); Da Costa (2012), reported that there were non-significant statistical differences in the measurements of right and left canines between males and females.

Results of premolars in the present study were in agreement with Babu et al. (2016) and Srinivasprasad et al. (2017), who found that the first premolars dimensions had significant degree of sexual dimorphism. On the other hand Kailash et al. (2018) found that there was statistical significant difference in $\mathrm{BL}$ and MD dimensions of the left first, right second and left second premolars between males and females.
Preeti et al. (1999); Rai et al. (2007) Ghodosi et al. (2008); Rai et al. (2008); Sonika et al. (2011) and Eboh (2012) found that there was significant degree of sexual dimorphism in right first molar dimensions which correlates with the results of the present study.

The present results came in contrast to Garn et al. (1967a) and Shireen and Ara (2016), who found that the right first molar dimensions didn't show any statistical significant difference between males and females.

On the other hand, the present results showed that the MD parameter of left first molar showed no statistical significant difference between males and females. Similar results were obtained by Suazo et al. (2008); Agnihotri and Sikri (2010); Kumar et al. (2016) and Narang et al. (2015) who found that MD dimension of left first molar had no role in sexual dimorphism.

Second molar findings of the present study were in agreement with Garn et al. (1966) who found that $\mathrm{BL}$ dimension showed greater degree of sexual dimorphism compared to MD diameter. The results of the current study were also in agreement with Sharma et al. (2013), who found that the MD dimension of right second molar exhibited greater sexual dimorphism and it was significantly predictive for male gender than BL dimension of the same tooth.

Staka et al. (2016) found that there was statistical significant difference as regard to central incisor indices and they had significant degree of prediction for male gender, this came in agreement with the results of the current study. In contrast to the present study, Introna et al. (1993) found that crown index of right central incisor and crown area only of the central incisors had high predicting value for male gender.

Canines findings of the current study were in agreement with İsçan and Kedici (2003) as 
well as Acharya and Mainali (2007), who proved that canine dental indices showed significant degree of sexual dimorphism. In contrast, Lund and Mörnstad (1999) and Karaman (2006) detected that the crown index of canines makes no contribution to sex dimorphism.

Ditch and Rose (1972) founded that right and left first premolars did not show statistical significant difference as regard to crown area or module between males and females. On the other hand, crown index showed significant degree of dimorphism and had significant predicting value for males, which came in agreement with the results of the current study. In contrast Garib and Peck (2006), found that crown index had significant predicting value for females.

Findings of molars in the current study are in agreement with Townsend and Brown, (1979), who noted that there was statistical significant difference in crown index values of first molars. Also in other studies performed by Rosenzweig (1970) and Garn et al. (1967b), the crown index for first molar was greater in females in a North American sample, the differences being statistically significant.

The current results regarding dental indices concluded that crown index presents a picture of sex dimorphism different to crown area, crown module and linear measurements. This was explained by Kondo and Townsend (2004) who found that crown index "indicates the relative size of (MD) and (BL) diameters, it expresses one linear measurement in terms of the other. While male linear dimensions are generally larger than females' in absolute terms, this may not be true when they are taken as a relative measure. Indeed, some consider crown index to be independent of the absolute values of linear dimensions (Rosenzweig, 1970).
The different patterns of sexual dental dimorphism observed between different populations reflect genetic and environmental influences to dental development (Srivastav et al., 2014). There are differences in odontometric features in specific populations, even within the same population in the historical and evolutionary context.

Differences in dimensions of the teeth are due to greater dentine thickness in males as compared to females, as the Y-chromosome increases the mitotic potential of the tooth germ and induces dentinogenesis (Garn et al. (1979) and Vodanovic et al. (2007).

\section{Conclusion}

The present study proved that BL and Crown index are the best parameters for assessment of gender in most of teeth of the upper jaw.

\section{References}

Acharya, A. B. and Mainali S. (2007): "Univariate sex dimorphism in the Nepalese dentition and the use of discriminate functions in gender assessment". Forensic Sci. Int., 173(1):4756.

Acharya, A.B. and Mainali, B. (2008):" Sex discrimination potential of buccolingual and mesiodistal tooth dimensions". J. Forensic Sci., 53(4):790.

Agnihotri, G. and Sikri, I. (2010): "Crown and cusp dimension of the maxillary first molar: a study of sexual dimorphism in Indian Jat Sikhs". Dental Anthropology, 23(1):1-6.

Al-Rifaiy, M. Q.; Abdullah, M. A.; Ashraf, I. and Khan, N. (1997): "Dimorphism of mandibular and maxillary canine teeth in establishing sex identity". Saudi Dent. J., 9(1):17-20. 
Astete, J. C.; San Pedro V. J. and Suazo G. I. (2009): "Sexual dimorphism in the tooth dimensions of Spanish and Chilean peoples". Int. J. Odontostomatol., 3(1):41-50.

Babu, S.S.; Nair, S.S.; Gopakumar, D.; Kurian, N. et al. (2016): "Linearodontometric analysis of permanent dentition as a forensic aid: A Retrospective study". J. Clin. Diagn. Res., 10: 24-28.

Bidmos, M.A.; Gibbon, V.E. and Štrkalj, G. (2010): "Recent advances in sex identification of human skeletal remains in South Africa". S. Afr. J. Sci., 106(11) 238:246.

Bakkannavar, S.M.; Monteiro, F.N.; Arun, M. and Kumar, P.G. (2012): "Mesiodistal width of canines: a tool for sex determination". Med. Sci. Law, 52(1): 22-26.

Da Costa, Y.T.F.; Lima, L.N.C. and Rabello, P.M. (2012):"Analysis of canine dimorphism in the estimation of sex". Braz. J. Oral. Sci., 11(3):406-410.

Ditch, L.E. and Rose J.C. (1972): "A multivariate dental sexing technique". Am. J. Phys. Anthropol.; 37:61.

Eboh, D.E. (2012): "Dimorphic study of maxillary first molar crown dimensions of Urhobos in Abraka". Journal of Morphological Sciences, 29(2):96-100.

Garib, D.G. and Peck S. (2006): "Extreme variations in the shape of maxillary premolars". Am. J. Orthod. Dentofacial Orthop., 130:317-323.

Garn, S.M.; Lewis, A.B. and Kerewsky, R.S. (1966):"Sexual dimorphism in the buccolingual tooth diameter". J. Dent. Res., 45:1819.
Garn, S.M.; Lewis, A.B. and Kerewsky, R.S. (1967a): "Buccolingual size asymmetry and its developmental meaning". Angle Orthod., 37:186-193.

Garn, S. M.; Lewis, A. B.; Swindler, D. R. \& Kerewsky, R. S. (1967b): "Sex difference in tooth shape". J. Dent. Res., 46:1470.

Garn, S. M.; Osborne, R. H. and McCabe, K. D. (1979): "The effect of prenatal factors on crown dimensions". Am. J. Phys. Anthropol., 51(4):665-678.

Ghodosi, A.; Mosharraf, R. and Nia, F.F. (2008): "Sexual variation in bucco-lingual dimensions in Iranian dentition". Int. J. Dent. Anthropol., 12:1-7.

İşcan, M.Y. and Kedici, P.S. (2003): "Sexual variation in bucco-lingual dimensions in Turkish dentition". Forensic Sci. Int., 137:160-164.

Introna, F. Jr.; Cantatore, F.; Dragone, M. and Colonna M. (1993): "Sexual dimorphism of deciduous teeth in medicolegal identification (abstract)". Boll. Soc. Ital. Biol. Sper., 69:223-230.

Kailash, C.D.; Abikshyeet, P.; Shyam, S. B.; et al. (2018): "Employing dimensional disparity of teeth to establish the gender in Odisha population: A dimorphic study". J. Int. Soc. Pre. Community Dent., 8 (2): 174-178.

Karaman F. (2006): "Use of diagonal teeth measurements in predicting gender in a Turkish population". J. Forensic Sci.; 51:630-635.

Kondo, S. and Townsend, G.C. (2004):" Sexual dimorphism in crown units of mandibular deciduous and permanent molars in Australian Aborigines". HOMO. J. Comparative Hum. Biol., 55: 53-64. 
Kumar, A.M.; Veena, K.M.; Chatra, L.; et al. (2016): "Gender predictability of permanent maxillary first molar, a south Indian study". Sch. Dent. Sci; 3:4-7.

Lund, H. and Mörnstad (1999): "Gender determination by odontometrics in a Swedish population". J. Forensic Odontotomatol., 17:30-34.

Nahidh, M. (2014): "The value of maxillary central incisors and canines in gender determination as an aid in forensic dentistry". Iraqi. Dent. J., 36(1):8-12.

Narang, R.S.; Manchanda, A.S. and Singh, B. (2015): "Sex assessment by molar odontometrics in North Indian population". J. Forensic Dent. Sci., 7:5458.

Omar, A. and Azab, S. (2009):"Applicability of determination of gender from odontometric measurements of canine teeth in a sample of adult Egyptian population". Cairo Dental Journal, 25(2):167-180.

Pereira, C.; Bernardo, M.; Pestana, D.; et al. (2010) : "Contribution of teeth in human forensic identification, Discriminant function sexing odontometrical techniques in Portuguese population". J. Forensic Leg. Med., 17(2):105-110.

Preeti, N.; Balaji, Rao, B. and Anniger, R.G. (1999): "A study of tooth size, symmetry and sexual dimorphism". J.F.M.T., 16:10-13.

Rai, B.; Jain, R.; Duhan, J.; Dutta, S. et al. (2007): "Importance of maxillary first molar for sex determination". Internet J. Dent. Sci., 4:2.
Rai, B.; Dhattarwal, S.K. and Anand, S.C. (2008): "Sex determination from tooth". Medico Legal Update, 8:3-5.

Ramakrishnan, K.; Sharma, S.; Sreeja, C.; et al. (2015):" Sex determination in forensic odontology: A review". J. Pharm. Bioall. Sci., 7: 398-402.

Rosenzweig, K.A. (1970): "Tooth form as a distinguishing trait between sexes and human populations". J .Dent. Res., 49:1423-1426.

Sabóia, T. M.; Tannure, P. N.; Luiz, R. R.; et al. (2013) :" Sexual dimorphism involved in the mesiodistal and buccolingual dimensions of permanent teeth". Dentistry 3000, 1(1), DOI 10.5195/d3000.2013.10.

Sharma, P.; Singh, T.; Kumar, P.C. and Sharma, R. (2013): "Sex determination potential of permanent maxillary molar widths and cusp diameters in a North Indian population". J. Orthod. Sci., 2(2):55-60.

Shireen, A. and Arshiya, S.A. (2016): "Odontometric analysis of permanent maxillary first molar in gender determination". J. Forensic Dent. Sci., 8(3):145-149.

Srivastava, R.; Jyoti, B.; Jha, P.; et al. (2014):" Gender determination from the mesiodistal dimension of permanent maxillary incisors and canines: An odontometric study". J.I.A.O.M.R., 26 (3):287-292.

Srinivasprasad, M.; Kattappagari, K.K.; Teja, C.S. et al. (2017): "Assessment of sexual dimorphism using odontometric analysis in the dentition of Guntur, South Indian population". Indian J. Foren. Med. Toxicol., 10:172-177. 
Sonika, V.; Harshaminder, K.; Madhushankari, G.S. et al. (2011): "Sexual dimorphism in the permanent maxillary first molar: A study of the Haryana population (India)". J. Forensic Odontostomatol., 29:37-43.

Staka, G. and Bimbashi, V. (2013): "Sexual dimorphism in permanent maxillary canines". Int. J. Pharm. Bio. Sci., 4(2):(B) $927-932$.

Staka, G.; Asllani, F. and Bimbashi, V. (2016): "Sexual dimorphism in permanent maxillary central incisor in Kosovo Albanian population". Int. J. Morphol., 34(3):1176-1180.

Suazo, G.I.; Cantin, L.M.; Lopez, F.B.; et al. (2008): "Sexual dimorphism in mesiodistal and bucolingual tooth dimensions in Chilean people". Int. J. Morphol., 26:609-614.

Townsend, G.C; and Brown, T. (1979): "Tooth size characteristics of Australian aborigines". Occas, Pap, Hum.Biol., 1:1738.

Vodanovic, M.; Demo, Z.; Njemirovskij, V.; et al. (2007) : "Odontometrics: A useful method for sex determination in an archaeological skeletal population". J. Archaeol. Sci., 34:905-913.

Zorba, E.; Moraitis, K. and Manolis, S.K. (2011): "Sexual dimorphism in permanent teeth of modern Greeks". Forensic Sci. Int., 15; 210(1-3):74-81. 


\section{التنبؤ بالجنس من الأبعاد الخطيه ومعاملات الاسنان}

\section{حسناء أحمد أحمد علي' ، رانيا. رضوان و مها هلال' \\ قسم الطب الثرعي و السموم الإكلينيكية، كلية الطب سوهاج - جامعه سوهاج}

تحديد الجنس هو أحد أهم الخطوات الحيوية في الطب الشرعي ويعتبر التمييز بين الجنسين عن طريق در اسه و تحليل قياسات الاسنان في مختلف الثعوب من العو امل التي يساعد في التعرف علي خصائص كل شعب وسهوله تمبيزه و تهدف هذه الدراسة الي إيجاد طريقه للتمييزبين الجنسيين عن طريق معاملات الأسنان

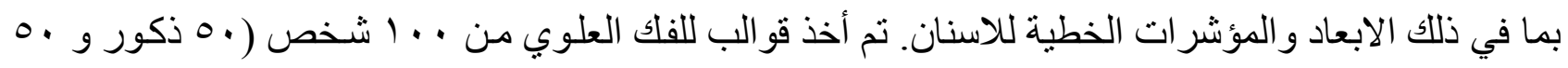

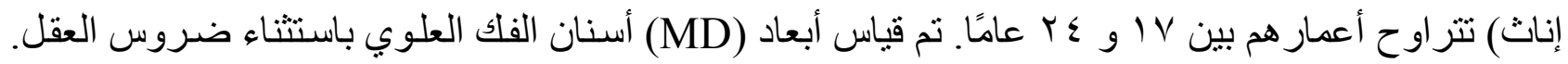

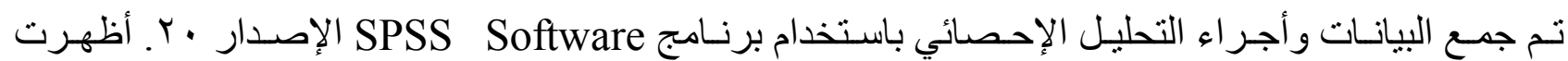
الدر اسة فروق ذات دلالة إحصائية بين الذكور والإناث في البعد الخطي ومؤشرات الأسنان في القو اطع الاماميـ برنه

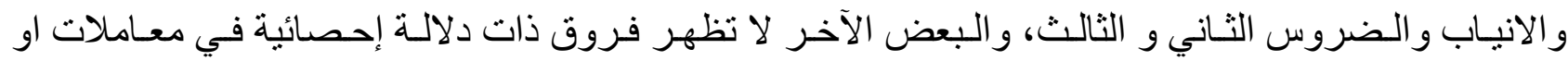
مؤشر ات الاسنان . 